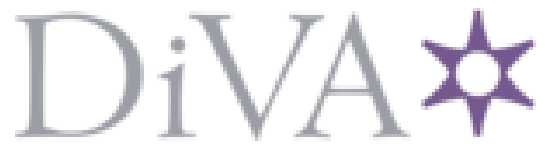

http://www.diva-portal.org

Preprint

This is the submitted version of a paper presented at IEEE/IAPR International Joint Conference on Biometrics, IJCB, Denver, Colorado, USA, October 1-4, 2017.

Citation for the original published paper:

Alonso-Fernandez, F., Farrugia, R., Bigun, J. (2017)

Learning-Based Local-Patch Resolution Reconstruction of Iris Smart-phone Images In: (pp. 787-793). New York: IEEE

https://doi.org/10.1109/BTAS.2017.8272771

N.B. When citing this work, cite the original published paper.

Permanent link to this version:

http://urn.kb.se/resolve?urn=urn:nbn:se:hh:diva-34687 


\section{Learning-Based Local-Patch Resolution Reconstruction of Iris Smart-phone Images}

\author{
Fernando Alonso-Fernandez \\ IS-Lab/CAISR \\ Halmstad University (Sweden) \\ feralo@hh.se
}

\author{
Reuben A. Farrugia \\ Department of CCE \\ University of Malta (Malta) \\ reuben.farrugia@um.edu.mt
}

\author{
Josef Bigun \\ IS-Lab/CAISR \\ Halmstad University (Sweden) \\ josef.bigun@hh.se
}

\begin{abstract}
Application of ocular biometrics in mobile and at a distance environments still has several open challenges, with the lack quality and resolution being an evident issue that can severely affects performance. In this paper, we evaluate two trained image reconstruction algorithms in the context of smart-phone biometrics. They are based on the use of coupled dictionaries to learn the mapping relations between low and high resolution images. In addition, reconstruction is made in local overlapped image patches, where upscaling functions are modelled separately for each patch, allowing to better preserve local details. The experimental setup is complemented with a database of 560 images captured with two different smart-phones, and two iris comparators employed for verification experiments. We show that the trained approaches are substantially superior to bilinear or bicubic interpolations at very low resolutions (images of $13 \times 13$ pixels). Under such challenging conditions, an EER of $\sim 7 \%$ can be achieved using individual comparators, which is further pushed down to 4-6\% after the fusion of the two systems.
\end{abstract}

\section{Introduction}

Iris is regarded as one of the most accurate biometric modalities available [12]. However, many applications which are becoming ubiquitous, such as smart-phone biometrics, have the lack of pixel resolution as one of their most evident problems that can jeopardize recognition accuracy when acquisition is done distantly or on the move [13]. In this context, super-resolution (SR) techniques can be used to enhance the quality of low resolution iris images to improve the recognition performance of existing systems. SR in biometrics is relatively recent, with research mainly concentrated in face reconstruction [23]. One reason of such limited research might be that most super-resolution approaches are general-scene, aimed at producing overall

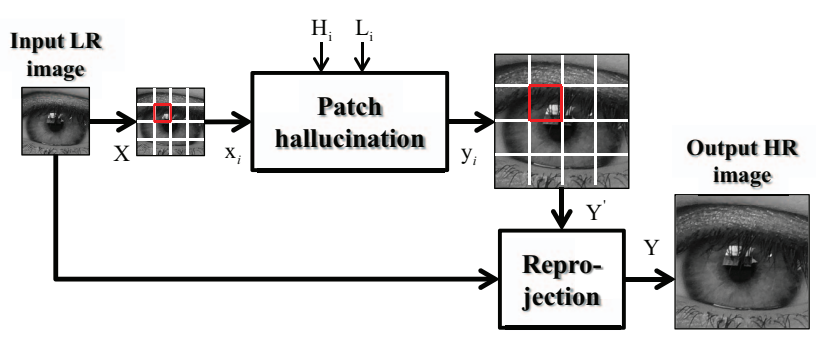

Figure 1. Block diagram of patch-based hallucination.

visual enhancement, which does not necessarily correlate with better recognition performance [18]. Thus, adaptation of super-resolution techniques to the particularities of images from a specific biometric modality is needed to achieve a more efficient up-sampling [6].

Two main categories of SR methods are distinguished in the literature: reconstruction- and learning-based [20]. Reconstruction-based methods fuse several low resolution (LR) images to obtain a high resolution (HR) image, with the disadvantage that multiple LR images are needed as input. Reconstruction-based methods to improve iris images from videos include for example the work [10], where authors compute the pixel-wise average of a number of aligned iris images, or the work [15], where authors apply PCA to unwrapped iris images in order to highlight the variance information among the pixel intensity vectors, and then compute the pixel-wise average of the resulting images. Both methods select as input images the frames with best quality from a given iris video stream. On the other hand, learning-based methods use coupled dictionaries to learn the mapping relations between LR and HR image pairs in order to hallucinate a HR image from the observed LR one. Learning-based methods have the advantage of only needing one image as input, and generally allows to achieve higher magnification factors [20]. Examples in iris recognition include for example [22], which uses Multi-Layer Perceptrons, or [9], which employs frequency analysis. A major limitation of these two learning-based works is that 


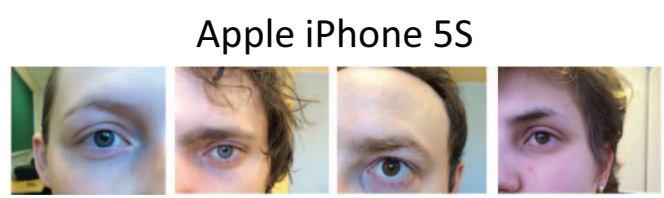

Nokia Lumia 1020

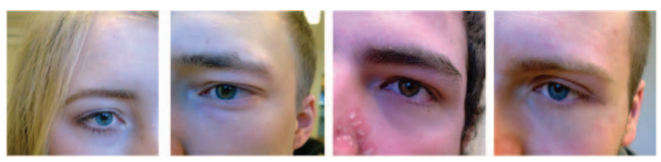

Figure 2. Sample images from VSSIRIS database [21].

they try to develop a prototype iris using combination of complete images. Patch-based methods, which models a local patch using collocated patches from the training dictionary, instead of using the whole image, have been also proposed. The work [11] for example employs Markov networks for this purpose, while the work [3] employs PCA. In these methods, each patch is hallucinated separately, having its own optimal reconstruction coefficients, which provides better quality reconstructed prototypes with better local detail and lower distortions. Local methods are also generally superior in recovering texture than global methods, which is essential due to the prevalence of texture-based methods in ocular biometrics [19].

This paper investigates two trained patch-based superresolution approaches in the context of smart-phone iris recognition. We evaluate the mentioned approach based on PCA Eigen transformation (eigen-patches) [3], and an implementation of the Locality-Constrained Iterative Neighbor Embedding (LINE) method [14] for iris images. We employ the Visible Spectrum Smart-phone Iris (VSSIRIS) database [21], captured with two smart-phones, with low resolution images having a size of only $13 \times 13$ pixels. Verification experiments are conducted with two iris comparators based on Log-Gabor wavelets [17] and SIFT keypoints [16]. Log-Gabor exploit texture information globally (across the entire iris image), while SIFT exploit local features (in discrete key points), therefore our motivation is to employ features that are diverse in nature, and reveal if they behave differently. Despite the LINE approach used is not new [14], we contribute with its implementation to smart-phone iris images, and particularly with the application (and fusion) of these two iris comparators to the reconstructed images. Reported results show the superiority of the two trained reconstruction approaches at very low resolutions w.r.t. bicubic or bilinear interpolations, with further reductions of more $\sim 30 \%$ in the EER due to the fusion of the two comparators.

\section{Low Resolution Iris Reconstruction}

Given an input low resolution (LR) image $\mathbf{X}$, the goal is to reconstruct its high resolution (HR) counterpart $\mathbf{Y}$. The LR image can be modeled as the HR image manipulated by blurring $(B)$, warping $(W)$ and down-sampling $(D)$ as $\bar{X}=D B W \bar{Y}+\bar{n}$ (where $\bar{n}$ represents additive noise). For simplicity, $W$ and $\bar{n}$ are usually omitted, leading to $\bar{X}=D B \bar{Y}$. In local patch-based methods (Figure 1), LR images are first separated into $N=N_{v} \times N_{h}$ overlapping patches $\mathbf{X}=\left\{\mathbf{x}_{1}, \mathbf{x}_{2}, \cdots, \mathbf{x}_{N}\right\}$ according to a predefined patch size and overlap pixels $\left(N_{v}\right.$ and $N_{h}$ are the vertical and horizontal number of patches). Since we will consider square images, we assume that $N_{v}=N_{h}$. Two super sets of basis patches $\mathbf{H}_{i}$ and $\mathbf{L}_{i}$ are computed for each patch $\mathbf{x}_{i}$ from collocated patches of a training database of $M$ high resolution images $\{\mathbf{H}\}$. The super set $\mathbf{H}_{i}=\left\{\mathbf{h}_{i}^{1}, \mathbf{h}_{i}^{2}, \cdots, \mathbf{h}_{i}^{M}\right\}$ is obtained from collocated patches of $\{\mathbf{H}\}$. By degradation (low-pass filtering and down-sampling), a low resolution database $\{\mathbf{L}\}$ is obtained from $\{\mathbf{H}\}$, and the other super set $\mathbf{L}_{i}=\left\{\mathbf{l}_{i}^{1}, \mathbf{l}_{i}^{2}, \cdots, \mathbf{l}_{i}^{M}\right\}$ is obtained similarly from $\{\mathbf{L}\}$. Each individual LR patch $\mathbf{x}_{i}$ is then hallucinated using the dictionaries $\mathbf{H}_{i}$ and $\mathbf{L}_{i}$, producing the corresponding HR patch $\mathbf{y}_{i}$.

\subsection{Eigen-Patch Reconstruction Method (PCA)}

This method is described in [3], which is based on the algorithm for face images of [7]. Here, a PCA eigen- transformation is conducted in the set of LR basis patches $\mathbf{L}_{i}$. Given an input LR patch $\mathbf{x}_{i}$, it is then projected onto the eigenpatches of $\mathbf{L}_{i}$, obtaining the optimal reconstruction weights $\mathbf{c}_{i}=\left\{c_{i}^{1}, c_{i}^{2}, \cdots, c_{i}^{M}\right\}$ of $\mathbf{x}_{i}$ w.r.t. $\mathbf{L}_{i}$. The reconstruction weights are then carried on to weight the HR basis set, and the HR patch is super-resolved as $\mathbf{y}_{i}=\mathbf{H}_{i} \mathbf{c}_{i}^{T}$. Finally, once the overlapping reconstructed patches $\left\{\mathbf{y}_{1}, \mathbf{y}_{2}, \cdots, \mathbf{y}_{N}\right\}$ are obtained, they are stitched together by averaging, resulting in the preliminary reconstructed $\mathrm{HR}$ image $\mathbf{Y}^{\prime}$.

\subsection{Locality-Constrained Iterative Neighbour Em- bedding Method (LINE)}

This method is based on the algorithm for face images of [14]. Here, instead of using all entries of the training dictionary to estimate the reconstruction weights, a smaller set of $K<M$ entries is used. Using all the entries can result in over-smooth reconstructed images which lacks important texture information, which is essential for iris. Given a LR patch $\mathbf{x}_{i}$, a first estimate of the HR patch $\mathbf{v}_{i, 0}$ is initialized by bicubic up-scaling. Then, an iterative loop indexed by $j \in[0, J-1]$ is started. For every iteration, the support $\mathbf{s}$ of $\mathbf{H}_{i}$ that minimizes the distance $\mathbf{d}=\left\|\mathbf{v}_{i, j}-\mathbf{H}_{i}(\mathbf{s})\right\|_{2}^{2}$ is computed using $K$-nearest neighbours. The combination weights are then derived using 


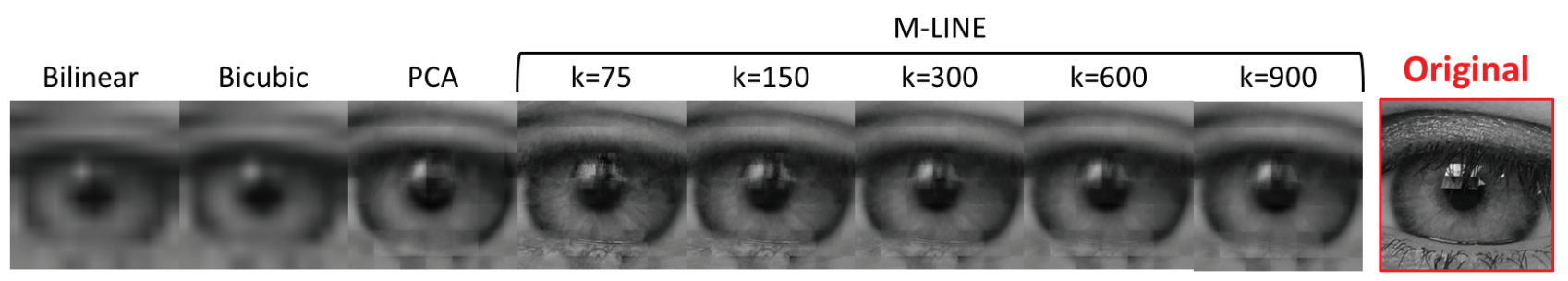

IPHONE

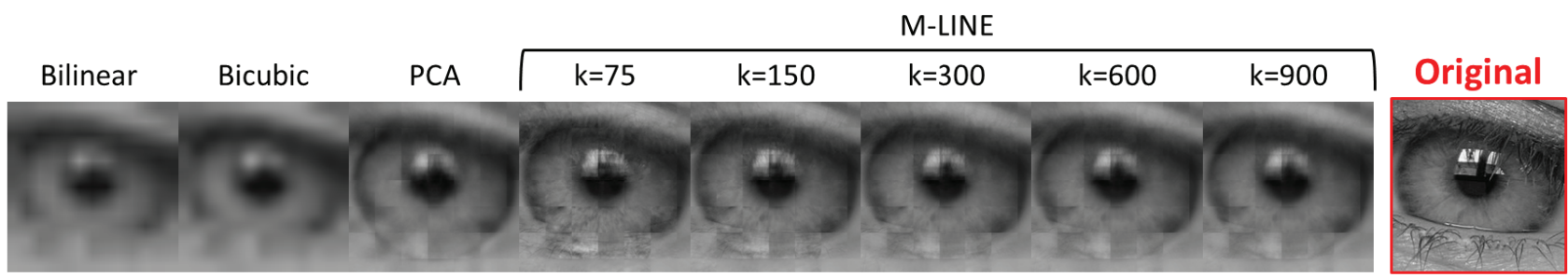

NOKIA

Figure 3. Resulting HR hallucinated images. The original HR image is also shown.

$$
\begin{gathered}
\mathbf{w}_{i, j}^{*}=\arg \min _{\mathbf{w}_{i, j}^{*}}\left(\left\|\mathbf{x}_{i}-\mathbf{L}_{i}(\mathbf{s}) \mathbf{w}_{i, j}^{*}\right\|_{2}^{2}+\right. \\
\left.+\tau\left\|\mathbf{d}(\mathbf{s}) \odot \mathbf{w}_{i, j}^{*}\right\|_{2}^{2}\right)
\end{gathered}
$$

where $\tau$ is a regularization parameter. Operator $\odot$ denotes the element-wise multiplication, and it is used to penalize the reconstruction weights with the distances between $\mathbf{v}_{i, j}$ and its closest neighbors in the training dictionary $\mathbf{H}_{i}$. This optimization problem can be solved by an analytic solution [14]. The estimated HR patch is then updated using $\mathbf{v}_{i, j+1}=\mathbf{H}_{i}(\mathbf{s}) \mathbf{w}_{i, j}^{\star}$ and the loop is repeated. The final estimate of the HR patch is then derived using $\mathbf{y}_{i}=\mathbf{v}_{i, J}$. We employ $\tau=1 e^{-5}$ and $J=4$ [14]. Contrarily to the PCA method, where reconstruction weights are obtained in the LR manifold and then simply transferred to the HR manifold, note that Equation 1 jointly considers the LR manifold (via $\mathbf{x}_{i}, \mathbf{L}_{i}(\mathbf{s})$ ) and the HR counterpart (via $\mathbf{d}(\mathbf{s})$ ) during the reconstruction. In addition, reconstruction starts in the HR manifold, which is not affected by the degradation process, and computation of the $K$ nearest neighbors employed for reconstruction is done in this manifold as well. On the other hand, the set of nearest neighbors with LINE is specific of a particular input patch $\mathbf{x}_{i}$, therefore it needs to be computed for each new patch. PCA on the contrary can be pre-trained in advance using the set $\mathbf{L}_{i}$ of basis patches, since eigen-patches are the same for any input patch $\mathbf{x}_{i}$, allowing faster computation times.

\subsection{Image Reprojection}

Inspired by [3], we incorporate a re-projection step to $\mathbf{Y}^{\prime}$ to reduce artifacts and make the output image $\mathbf{Y}$ more similar to the input image $\mathbf{X}$. The image $\mathbf{Y}^{\prime}$ is re-projected to $\mathbf{X}$ via $\mathbf{Y}^{t+1}=\mathbf{Y}^{t}-v U\left(B\left(D B \mathbf{Y}^{t}-\mathbf{X}\right)\right)$ where $U$ is the up-sampling matrix. The process stops when $\mid \mathbf{Y}^{t+1}-$ $\mathbf{Y}^{t} \mid \leq \varepsilon$. We use $v=0.02$ and $\varepsilon=10^{-5}$ [3].

\section{Experimental Framework}

\subsection{Dataset}

We use the Visible Spectrum Smart-phone Iris (VSSIRIS) database [21], which consists of images from 28 subjects (56 eyes) captured using the rear camera of two different smart-phones (Apple iPhone 5S and Nokia Lumia 1020). Images from the iPhone $5 S$ have $3264 \times 2448$ pixels, while images from the Lumia 1020 have $3072 \times 1728$ pixels. Images have been obtained in unconstrained conditions under mixed illumination consisting of natural sunlight and artificial room light. Each eye has 5 samples per smart-phone, thus totalling $5 \times 56=280$ images per device ( 560 in total). Acquisition is done without flash, in a single session and with semi-cooperative subjects. Figure 2 shows some example images. Iris segmentation data is also available, which has been used as input for our experiments. All images of the database have been resized via bicubic interpolation to have the same sclera radius (we choose as target radius the average sclera radius $R=145$ of the whole database, given by the groundtruth). Then, images are aligned by extracting a square region of $319 \times 319$ around the sclera center (corresponding to about $1.1 \times R$ ). Two sample images can be seen in Figure 3, right.

Aligned and normalized HR iris images are then downsampled via bicubic interpolation to a size of $13 \times 13$, corresponding to a down-sampling factor of 22 , and then used 
as input LR images of the reconstruction methods, from which hallucinated HR images are computed. This simulated down-sampling is the approach followed in most previous studies [23], mainly due to the lack of databases with LR and corresponding HR reference images. Given an input LR image, we use all available images from the remaining eyes (of both smart-phones) to train the hallucination methods (leave-one-out). Training images are mirrored in the horizontal direction to duplicate the size of the training dataset, thus having 55 eyes $\times 10$ samples $\times 2=1100$ images for training. In PCA and LINE, we employ a patch size of $1 / 4$ of the LR image size. This is motivated by [2], where better results were obtained with bigger patch sizes. Overlapping between patches is $1 / 3$ of the patch size. We also compare our method with bicubic and bilinear interpolation. Figure 3 shows an example of hallucinated images. We test the LINE method using different values of $K$, from $K=75$ (small neighbors set) to $K=900$ (nearly the whole training set). It can be observed that smaller values of $K$ produces sharper reconstructed images, while a bigger $K$ produces blurrier images. This is expected, since a bigger value of $K$ implies that more patches are being averaged, so the output image patch will be smoother.

\subsection{Verification Experiments}

We conduct iris recognition experiments using two different systems based on 1D Log-Gabor filters (LG) [17] and the SIFT operator [16]. In LG, the iris region is first unwrapped to a normalized rectangle of $20 \times 240$ pixels [8] and next, a 1D Log-Gabor wavelet is applied plus phase binary quantization to 4 levels. Comparison between binary vectors is done using the normalized Hamming distance [8]. In the SIFT method, SIFT key points are directly extracted from the iris region (without unwrapping), and the recognition metric is the number of matched key points, normalized by the average number of detected key-points in the two images under comparison. The LG implementation is from Libor Masek [17], using its default parameters. The SIFT method uses a free toolkit ${ }^{1}$, with adaptations described in [5] to remove spurious matchings. The iris region and corresponding noise mask for feature extraction and matching is obtained from the available annotation of the database.

Verification experiments are as follows. Experiments are

${ }^{1}$ http://vision.ucla.edu/ vedaldi/code/sift/assets/sift/index.html

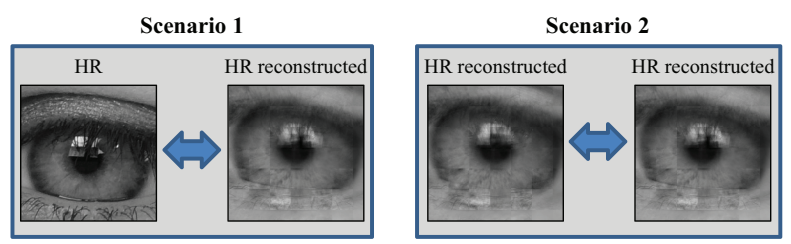

Figure 4. Scenarios considered in our experiments. done separately for each smart-phone. We consider each eye as a different user. Genuine trials are obtained by comparing each image of an eye to the trials images of the same eye, avoiding symmetric matches. Impostor matches are obtained by comparing the $1^{\text {st }}$ image of an eye to the $2^{\text {nd }}$ image of the remaining eyes. With this procedure, we obtain $56 \times 10=560$ genuine and $56 \times 55=3,018$ impostor scores per smart-phone.

\section{Results}

The performance of the hallucination algorithms is measured by reporting verification experiments using hallucinated HR images. We do not employ other reconstruction measures traditionally used in super-resolution literature (such as the Peak Signal-to-Noise Ratio or the Structural Similarity index between the hallucinated HR image and the corresponding HR reference image). These generalscene indicators measure the overall visual enhancement, and they does not necessarily correlate with better recognition performance, which is the aim of applying SR algorithms in biometrics [18]. As reported later, resolution reduction has not the same impact on the two biometric comparators employed, therefore general-scene indicators are not useful in our context.

We consider two scenarios in our experiments (Figure 4): 1) enrolment samples taken from original HR input images, and query samples from hallucinated HR images; and 2) both enrolment and query samples taken from hallucinated HR images. The first case simulates a controlled enrolment scenario, while the second case simulates a totally uncontrolled scenario (albeit for simplicity, both samples have similar resolution). Verification results are given in Table 1 , where it can be seen the advantages of using LINE at very low resolutions (LR image size is of $13 \times 13$ in our experiments). PCA also shows better performance in general than bilinear/bicubic interpolations, highlighting the benefits of trained reconstruction algorithms. Concerning the performance of the individual comparators, despite SIFT is much better than $\mathrm{LG}$ at high resolution $(<1 \%$ vs. $\sim 8 \%)$, its performance decreases dramatically at low resolution. While performance of LG decreases only between 1 and 3\%, SIFT worsens one or two orders of magnitude.

It is also worth noting the significantly better performance of scenario 2 with both comparators and both sensors. In this scenario, both images are subjected to the same down-sampling and reconstruction procedure, while in scenario 1 , the gallery image employed is the original HR image. This suggest that the information recovered by the reconstruction algorithms does not fully resemble the information found in the original HR image, at least as measured by the feature extractors employed here. Indeed, the performance of both comparators is less affected in relative numbers in scenario 2 . Regarding the neighborhood size $K$ 
IPHONE



Table 1. Verification results (EER) of the different reconstruction methods employed. Results with original high resolution images only are also shown as reference (row 'HR').

of LINE, there are no conclusive results. In scenario 1, the comparators have opposite behavior (LG prefers a bigger set, while SIFT prefers a small set). On the other hand, in scenario 2, both comparators tend to choose a bigger set. As a general rule, in most columns, the best results are obtained with $K \geq 600$; and in the remaining cases, performance is not significantly affected if we choose $K=300$.

We then carry out fusion experiments using linear logistic regression. Given $N$ comparators ( $N=2$ in our case) which output the scores $\left(s_{1 j}, s_{2 j}, \ldots s_{N j}\right)$ for an input trial $j$, a linear fusion is: $f_{j}=a_{0}+a_{1} \cdot s_{1 j}+a_{2} \cdot s_{2 j}+\ldots+a_{N} \cdot s_{N j}$. The weights $a_{0}, a_{1}, \ldots a_{N}$ are trained via logistic regression as described in [4]. We use this trained fusion approach because it has shown better performance than simple fusion rules (like the mean or the sum rule) in previous works. Results are given in Table 2. As it can be observed, the fusion of the two comparators provide an additional improvement. This is specially relevant in scenario 2, where EER reductions of more than $30 \%$ are obtained using LINE, pushing the EER to $4.1 \%$ (iphone) and 5.8\% (nokia). We further observe the benefit of LINE w.r.t. the other methods in Figure 5, where we report the DET curves of the individual comparators and of the fusion (scenario 2 only). The performance of PCA (green curves) is systematically outperformed by LINE in nearly any FAR/FRR region. It can be also appreciated in the DET curves that, in general, a bigger neighborhood is preferred by LINE $(K>150)$.

\section{Conclusion}

More relaxed acquisition environments are pushing ocular biometrics towards the use of low resolution imagery and sensors in the visible spectrum [1, 19]. This can pose significant problems in terms of reduced performance if not tackled properly. This paper addresses the problem of re- construction of low resolution iris images captured with smart-phones. We apply two trained super-resolution approaches based on PCA transformation [2] and LocalityConstrained Iterative Neighbor Embedding (LINE) of local patches [14] to improve the resolution of iris images. We also carry out verification experiments on the reconstructed images using two iris comparators based on Log-Gabor (LG) wavelets and SIFT key-points. Two operational scenarios are considered, one where original high-resolution images are matched against hallucinated high-resolution images (controlled enrolment), and another scenario where only hallucinated images are used (uncontrolled scenario). Low resolution images are simulated by down-sampling high-resolution irises to a size of just $13 \times 13$. Our experiments show the benefits of using trained approaches w.r.t. bilinear or bicubic up-sampling under such challenging conditions, with the LINE method showing additional superiority. This can be attributed to the joint use of the LR and HR manifolds during the reconstruction in LINE, since the low and high resolution dictionaries are coupled for the estimation of the reconstruction weights. PCA, on the other hand, obtains the reconstruction weights only in the LR manifold, and they are simply transferred to the HR manifold. As a result, better performance is provided by LINE.

The two trained reconstruction methods evaluated here assume that the low and high resolution manifolds have similar local geometrical structure (reflected by the use of the same reconstruction weights in both manifolds). This simplification is not usually the case, since the degradation suffered by low resolution images results in one-to-many relationship between low and high resolution patches, and it will be an avenue of future work [23]. Another simplifying approach that we will seek to overcome is the assumption 
IPHONE

\begin{tabular}{ll} 
scenario 1 & scenario 2 \\
\hline LG+SIFT & LG+SIFT
\end{tabular}

HR $(319 \times 319)$

\begin{tabular}{|l|l|}
\hline $0.03 \%$ & $0.03 \%$ \\
\hline
\end{tabular}

bilin
bicub
PCA

LINE $\mathrm{k}=75$

LINE $\mathrm{k}=150$

LINE $\mathrm{k}=300$

LINE $\mathrm{k}=600$

LINE k=900

\begin{tabular}{|c|c|}
\hline $18.46 \%$ & $8.99 \%$ \\
\hline $17.85 \%$ & $9.66 \%$ \\
\hline $10.12 \%$ & $4.64 \%$ \\
\hline $10.19 \%$ & $6.97 \%$ \\
$10.42 \%$ & $5.31 \%$ \\
$\mathbf{9 . 1} \%$ & $4.46 \%$ \\
$9.64 \%$ & $\mathbf{4 . 1} \%$ \\
$9.81 \%$ & $4.69 \%$ \\
\hline
\end{tabular}

NOKIA

\begin{tabular}{ll} 
scenario 1 & scenario 2 \\
\hline LG+SIFT & LG+SIFT \\
\hline
\end{tabular}

\begin{tabular}{|l|l|}
$0.34 \%$ & $0.34 \%$ \\
\hline
\end{tabular}

\begin{tabular}{|c|c|}
\hline $15.96 \%$ & $8.03 \%$ \\
\hline $14.74 \%$ & $8.23 \%$ \\
\hline $10.32 \%$ & $7.53 \%$ \\
\hline $11.7 \%$ & $6.94 \%$ \\
$10.15 \%$ & $6.19 \%$ \\
$9.38 \%$ & $5.87 \%$ \\
$\mathbf{8 . 7 3} \%$ & $\mathbf{5 . 7 8} \%$ \\
$9.61 \%$ & $6.58 \%$ \\
\hline
\end{tabular}

Table 2. Fusion results (EER) of the LG and SIFT comparators. Results with original high resolution images only are also shown as reference (row 'HR').
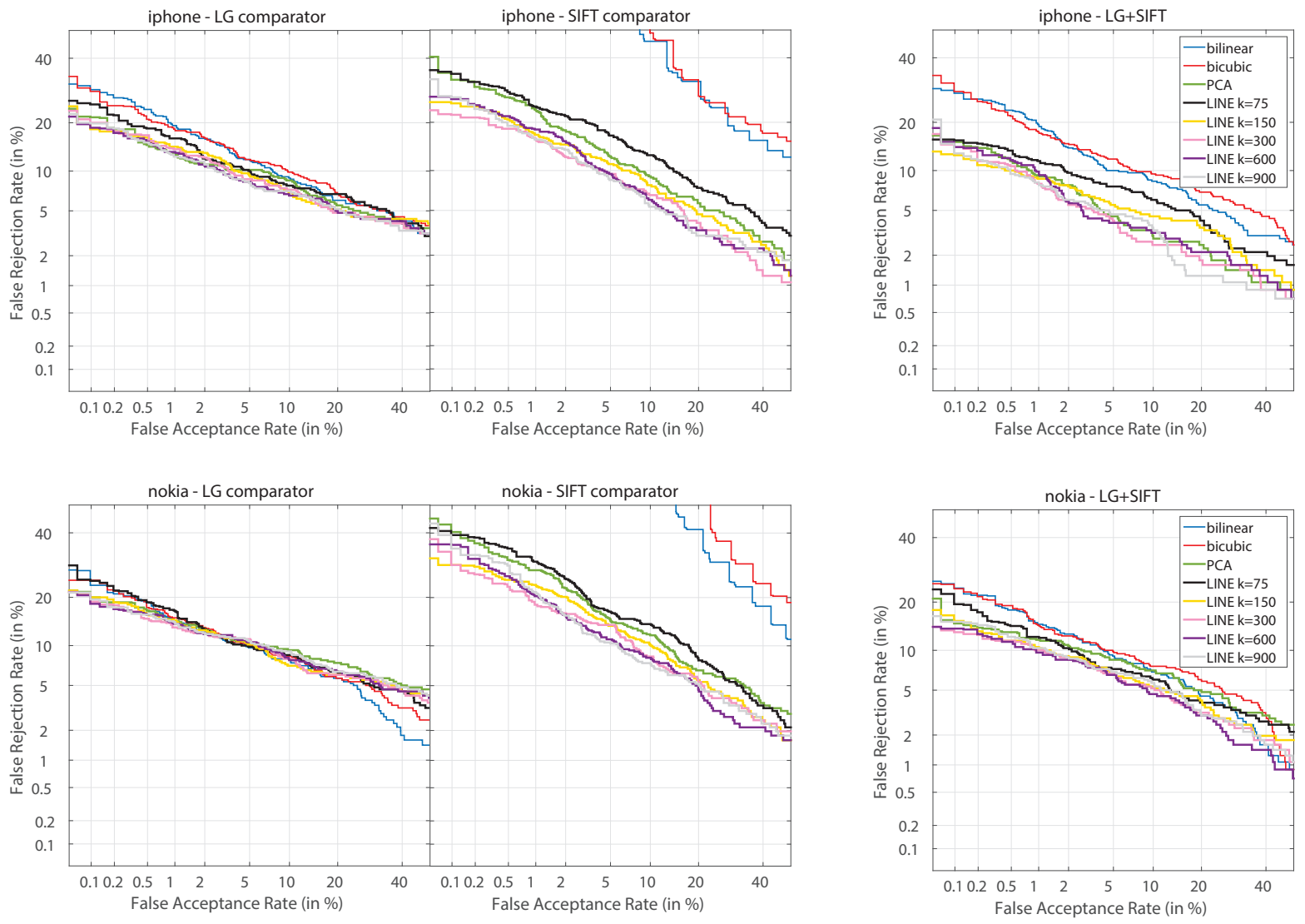

Figure 5. Fusion results (DET curves). Results are given for scenario 2 and LR image size of $13 \times 13$ only. Best seen in color.

of linearity in the combination of patches from the training dictionary. Another direction includes the use of strategies to cope with misalignments of the eye, since real low resolution images usually have blurring, and so many ambiguities will exist for accurate eye localization.

\section{Acknowledgments}

Author F. A.-F. thanks the Swedish Research Council for funding his research. Authors acknowledge the CAISR program and the SIDUS-AIR project of the Swedish Knowledge Foundation. 


\section{References}

[1] F. Alonso-Fernandez and J. Bigun. A survey on periocular biometrics research. Pattern Recognition Letters, 82:92-105, 2016.

[2] F. Alonso-Fernandez, R. A. Farrugia, and J. Bigun. Eigenpatch iris super-resolution for iris recognition improvement. Proc European Signal Processing Conference, EUSIPCO, Sep 2015.

[3] F. Alonso-Fernandez, R. A. Farrugia, and J. Bigun. Reconstruction of smartphone imges for low resolution iris recognition. Proc International Workshop on Information Forensics and Security, WIFS, Nov 2015.

[4] F. Alonso-Fernandez, J. Fierrez, D. Ramos, and J. GonzalezRodriguez. Quality-based conditional processing in multibiometrics: Application to sensor interoperability. IEEE Trans. on Systems, Man and Cybernetics-Part A: Systems and Humans, 40(6):1168-1179, 2010.

[5] F. Alonso-Fernandez, P. Tome-Gonzalez, V. Ruiz-Albacete, and J. Ortega-Garcia. Iris recognition based on sift features. Proc IEEE Intl Conf Biometrics, Identity and Security, BIDS, 2009.

[6] S. Baker and T. Kanade. Limits on super-resolution and how to break them. Pattern Analysis and Machine Intelligence, IEEE Transactions on, 24(9):1167-1183, Sep 2002.

[7] H.-Y. Chen and S.-Y. Chien. Eigen-patch: Position-patch based face hallucination using eigen transformation. In Multimedia and Expo (ICME), 2014 IEEE Intl Conf, pages 1-6, Jul 2014.

[8] J. Daugman. How iris recognition works. IEEE Trans. on Circuits and Systems for Video Technology, 14:21-30, 2004.

[9] A. Deshpande, P. P. Patavardhan, and D. H. Rao. Superresolution for iris feature extraction. Proc Intl Conf on Computational Intelligence and Computing Research, ICCIC, pages 1-4, 2014.

[10] K. Hollingsworth, T. Peters, K. Bowyer, and P. Flynn. Iris recognition using signal-level fusion of frames from video. IEEE Transactions on Information Forensics and Security, 4(4):837-848, 2009.

[11] J. Huang, L. Ma, T. Tan, and Y. Wang. Learning based resolution enhancement of iris images. Proc. BMVC, 2003.

[12] A. Jain, P. Flynn, and A. Ross, editors. Handbook of Biometrics. Springer, 2008.

[13] A. K. Jain and A. Kumar. Second Generation Biometrics, chapter Biometrics of Next Generation: An Overview. Springer, 2010.

[14] J. Jiang, R. Hu, Z. Wang, and Z. Han. Face super-resolution via multilayer locality-constrained iterative neighbor embedding and intermediate dictionary learning. IEEE Transactions on Image Processing, 23(10):4220-4231, Oct 2014.

[15] R. Jillela, A. Ross, and P. Flynn. Information fusion in lowresolution iris videos using principal components transform. Proc. IEEE Workshop on Applications of Computer Vision, WACV, pages 262-269, Jan 2011.

[16] D. Lowe. Distinctive image features from scale-invariant key points. Intl Journal of Computer Vision, 60(2):91-110, 2004.

[17] L. Masek. Recognition of human iris patterns for biometric identification. Master's thesis, School of Computer Science and Software Engineering, University of Western Australia, 2003.

[18] K. Nguyen, S. Sridharan, S. Denman, and C. Fookes. Feature-domain super-resolution framework for gabor-based face and iris recognition. In Proc IEEE Conf on Computer Vision and Pattern Recognition, CVPR, pages 2642-2649, Jun 2012.

[19] I. Nigam, M. Vatsa, and R. Singh. Ocular biometrics: A survey of modalities and fusion approaches. Information $\mathrm{Fu}$ sion, 26(0): 1 - 35, 2015.

[20] S. C. Park, M. K. Park, and M. G. Kang. Super-resolution image reconstruction: a technical overview. Signal Processing Magazine, IEEE, 20(3):21-36, May 2003.

[21] K. B. Raja, R. Raghavendra, V. K. Vemuri, and C. Busch. Smartphone based visible iris recognition using deep sparse filtering. Pattern Recognition Letters, 57:33-42, 2015.

[22] K. Y. Shin, K. R. Park, B. J. Kang, and S. J. Park. Superresolution method based on multiple multi-layer perceptrons for iris recognition. In Intl Conf Ubiquitous Information Technologies Applications, ICUT, pages 1-5, Dec 2009.

[23] N. Wang, D. Tao, X. Gao, X. Li, and J. Li. A comprehensive survey to face hallucination. Intl Journal of Computer Vision, 106(1):9-30, 2014. 